\title{
Venous super-drained posterior interosseous artery flap for dorsal hand defects
}

\section{Erhan Sönmez MD | Ersin Aksam MD ๑ | Mustafa Durgun MD | Onder Karaaslan MD}

Department of Plastic, Reconstructive and Aesthetic Surgery, Izmir Katip Celebi University, Faculty of Medicine, Ataturk Training and Research Hospital, İzmir, Turkey

\section{Correspondence}

Ersin Aksam, Izmir Katip Celebi Universitesi, Ataturk Egitim ve Arastirma Hastanesi, Plastik Cerrahi Klinigi, Izmir, Turkey, 35150.

Emails: ersinaksam@gmail.com;

drokaraaslan@yahoo.com

\begin{abstract}
Background: Posterior interosseous artery flap PIAF is one of the most preferred reconstructive options for upper extremity defects. As a reverse flap, venous congestion is always a threatening factor. In this report authors aimed to present their experience with venous super-drained posterior interosseous artery flap VS-PIAF.

Methods: Between January 2013 to September 17, 2017 patients were treated with VS-PIAF. Tumor excision, trauma, traffic accident, burn contracture release, and industrial injuries were the causes of defects. Defects were located on the dorsum of the hand, first web space, ulnar and radial side of the hand, and the palm. All the defects were reconstructed with a VS-PIAF, which a distal superficial end-to-end venous anastomosis was added to overcome venous congestion problems.

Results: The flap sizes were between $5 \times 7 \mathrm{~cm}$ and $8 \times 12 \mathrm{~cm}$. All the flaps have survived completely without any early or late complications. No venous problems were seen. The donor areas were healed uneventfully. The mean follow-up time was 15.3 months. The results of reconstructions were satisfactory, no functional restrictions was seen.

Conclusion: The major drawback of PIAF is venous congestion problems that can be manageable with adding a distal superficial end-to-end venous anastomose. VS-PIAF is a robust, much safer and easy-to-learn reconstructive method for upper extremity defects.
\end{abstract}

\section{1 | INTRODUCTION}

Reconstruction of upper extremity soft tissue defects should be done with pliable and thin tissues to preserve hand functions and mobility. Several methods including skin grafts and local and distant flaps have been described for reconstruction of hand and wrist defects. However, skin grafts have minimal usage in hand and wrist reconstruction because of secondary contracture. Using flap tissues is much more appropriate than skin grafts. Distant flaps such as abdominal interpolation flaps require two-stage operations, and there is discomfort to patients between these operations. Free flaps are the final stage of the reconstruction ladder and should be reserved for patients for whom no other reconstructive options remain. However, some authors prefer the concept of reconstructive elevator which suggests to use free flaps as the first choice if it will give the best result for the patient.

The posterior interosseous artery flap PIAF, which is one of the local flaps that is available, was first described by Zancolli and Angrigiani in 1988 (Zancolli \& Angrigiani, 1988). The PIAF is a reverse flow fasciocutaneous flap that is nourished through perforating arteries of the posterior interosseous artery. This flap can be used to reconstruct wrist and hand defects and even finger defects that are near to the distal interphalangeal joint level. The PIAF has become popular because it offers some great advantages, such as protecting the major arteries of the hand and having a long pedicle, a robust blood supply, and fewer problems in the donor area. The major disadvantage of the PIAF is venous congestion problems that can occur because it is a reverse flap (Balakrishnan, Kumar, \& Hussain, 2003; Brunelli, Valenti, Dumontier, Panciera, \& Gilbert, 2001; Dogra, Singh, Chakravarty, \& Basu, 2006; Ege, Tuncay, \& Ercetin, 2003; Lu, Gong, Lu, \& Wang, 2007). Many modifications have been described to overcome these venous problems, including using a wider pedicle to raise the flap, adding a fascia to the pedicle, adding a superficial vein to the pedicle, and making venous anastomoses (Acharya, Bhat, \& Bhaskaranand, 2012; Fong \& Chew, 2014; Ozalp, Elbey, Aydin, \& Ozkan, 2016; Yazar, Chen, \& Mardini, 2008). In this report, we present our experience with venous super-drained posterior interosseous artery flaps VS-PIAF for 
reconstruction of hand defects of 17 patients, which we performed using a distal end-to-end superficial venous anastomosis to overcome venous congestion problems.

\section{2 | PATIENTS AND METHODS}

This report included 17 patients who had upper extremity defects and had been treated with VS-PIAFs from January 2013 to September 2017. The mean age of the patients was 36.7 range $18-59$ years. Six of the patients were female, while 11 were male. The most common cause of the tissue defects was tumor excision five patients, and other causes were trauma four patients, traffic accidents three patients, burn contracture releases three patients, and industrial injuries two patients. Patient demographics were listed in Table 1. The defects were located on the dorsum of the hand, the first web space, ulnar, and radial side of the hand, and the palm. Four of the patients had comorbidities, including hypertension, diabetes mellitus, and chronic obstructive lung disease. All patients received anti-coagulation therapy $60 \mathrm{mg}$ of enoxaparin sodium for 3 days postoperatively.

\section{3 | SURGICAL TECHNIQUE}

The dimensions of the defect in each patient were noted. A straight line was drawn from the lateral epicondyle to the ulnar head, which represents the route of the posterior interosseous artery. A point that was $2 \mathrm{~cm}$ proximal from the distal radio-ulnar joint on this line was marked as the turning point of the flap. Flap markings were done according to predefined markings and with the aid of a gauze pad to simulate rotation of the flap (Figures $1 \mathrm{~A}$ and $2 \mathrm{~B}$ ). No Doppler ultrasound was used to determine the perforating arteries. A tourniquet was applied, but no Esmarch bandages were used to drain the blood away. The dissection of the veins was much easier when there was some remaining blood. As this kind of a "reverse-flow flap," planning of the flap and the nomenclature can be confusing. The operations were started with superficial incisions on the proximal part of the forearm to determine and dissect superficial veins that will drain distal part of the flap (Figures $1 \mathrm{~B}$ and $2 \mathrm{~B}$ ). After defining superficial veins that were appropriate for venous anastomosis, flap elevation was started from the ulnar side of the flap. The muscle belly of the extensor carpi ulnaris muscle was reached, and the deep fascia was incised. The dissection underneath the deep fascia was continued through the radial side until it reached the perforators coming from the posterior interosseous artery. The radial side of the flap was then incised through the deep fascia of the extensor digiti minimi muscle, and the flap was raised underneath the deep fascia to the ulnar side until it reached the perforators. A lazy-S incision was made from the proximal part of the flap to a predefined turning point. Bilateral skin flaps were elevated preserving the subdermal vascular plexus. Flap elevation was started by dividing the vessel in the most distal part of the flap and then raising the flap, including the intermuscular septum and deep fascia. The dissection was continued until there was an adequate pedicle length, and care was taken not to harm the fascial plexus and the

TABLE 1 Patient demographics

\begin{tabular}{|c|c|c|c|c|c|c|c|c|c|}
\hline $\begin{array}{l}\text { Patient } \\
\text { no }\end{array}$ & Age & Gender & Cause & Co-morbidities & $\begin{array}{l}\text { Location } \\
\text { of defect }\end{array}$ & $\begin{array}{l}\text { Flap } \\
\text { dimensions } \\
\text { cm }\end{array}$ & Complications & $\begin{array}{l}\text { Follow up } \\
\text { time } \\
\text { months }\end{array}$ & $\begin{array}{l}\text { Final } \\
\text { outcome }\end{array}$ \\
\hline 1 & 28 & $M$ & Traumatic & & Ulnar side & 610 & - & 24 & Satisfactory \\
\hline 2 & 36 & $M$ & Traumatic & & First web & $8 \times 12$ & - & 26 & Satisfactory \\
\hline 3 & 24 & $M$ & $\begin{array}{l}\text { Burn } \\
\text { contracture }\end{array}$ & & Palm & $5 \times 8$ & - & 23 & Satisfactory \\
\hline 4 & 18 & $\mathrm{~F}$ & Industrial injury & & $\begin{array}{l}\text { Radial } \\
\text { side }\end{array}$ & $5 \times 9$ & - & 18 & Satisfactory \\
\hline 5 & 47 & $M$ & Tumor & $\mathrm{HT}$ & Ulnar side & $5 \times 13$ & - & 20 & Satisfactory \\
\hline 6 & 49 & $\mathrm{~F}$ & Traffic accident & DM & Dorsum & $7 \times 10$ & - & 16 & Satisfactory \\
\hline 7 & 33 & $M$ & Tumor & & Dorsum & $5 \times 7$ & - & 19 & Satisfactory \\
\hline 8 & 59 & $M$ & Tumor & $\begin{array}{l}\text { HT, DM, } \\
\text { COLD }\end{array}$ & Dorsum & $5 \times 10$ & - & 16 & Satisfactory \\
\hline 9 & 21 & $\mathrm{~F}$ & Traumatic & & $\begin{array}{r}\text { Radial } \\
\text { side }\end{array}$ & $7 \times 11$ & - & 12 & Satisfactory \\
\hline 10 & 35 & $M$ & $\begin{array}{l}\text { Burn } \\
\text { contracture }\end{array}$ & & Dorsum & $5 \times 8$ & - & 14 & Satisfactory \\
\hline 11 & 44 & $M$ & Tumor & & Dorsum & $8 \times 10$ & - & 17 & Satisfactory \\
\hline 12 & 40 & $M$ & Industrial injury & & Palm & $7 \times 10$ & - & 11 & Satisfactory \\
\hline 13 & 31 & $\mathrm{~F}$ & Traffic accident & & Ulnar side & $6 \times 11$ & - & 13 & Satisfactory \\
\hline 14 & 38 & $M$ & Traffic accident & & Dorsum & $8 \times 12$ & - & 10 & Satisfactory \\
\hline 15 & 42 & $\mathrm{~F}$ & Traumatic & & Ulnar side & $6 \times 10$ & - & 9 & Satisfactory \\
\hline 16 & 53 & $M$ & Tumor & HT, DM & Dorsum & $6 \times 9$ & - & 7 & Satisfactory \\
\hline 17 & 26 & $\mathrm{~F}$ & $\begin{array}{l}\text { Burn } \\
\text { contracture }\end{array}$ & & Dorsum & $7 \times 12$ & - & 6 & Satisfactory \\
\hline
\end{tabular}



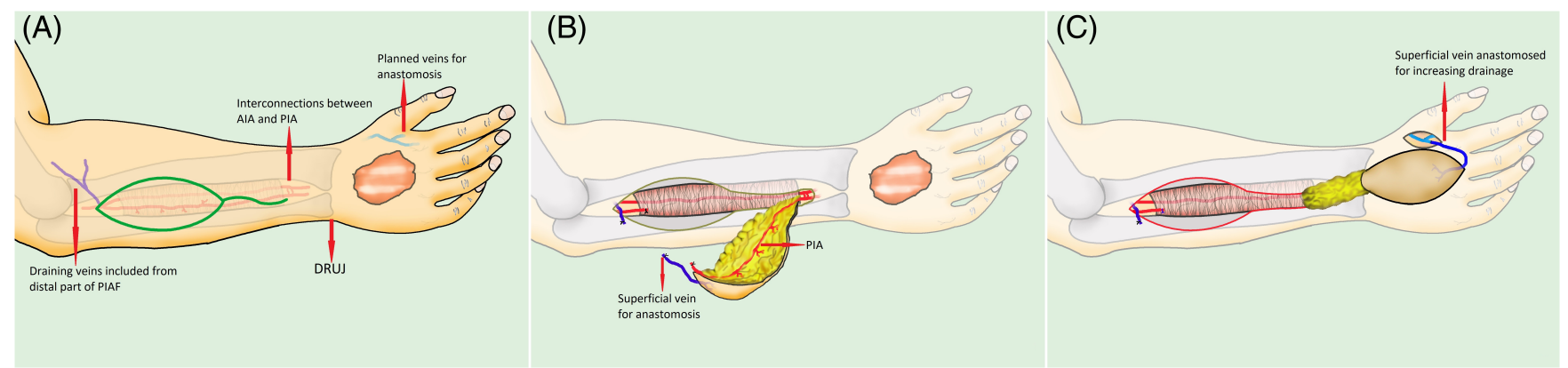

FIGURE 1 A, The schematic illustration of the VS-PIAF design. B, The schematic illustration of the elevation of VS-PIAF. C, The schematic illustration of the adaptation of VS-PIAF and venous anastomose

branch to the distal ulnar. A wide tunnel was formed to protect the vascular pedicle from pressure. The flap was then tunneled under the skin bridge to the defect, and a superficial vein was dissected for venous anastomosis. End-to-end venous anastomosis was done under loupe magnification $4 \times$ loupes with $9 / 0$ polyprolene sutures (Figures $1 \mathrm{C}$ and $2 \mathrm{C}$ ). The patency of the anastomosis was tested using a refill test. The remaining part of the nonanastomosed vein in the flap tissue was cauterized, and the flap was adapted with subdermal and cutaneous sutures (Figure 2D). The flap donor area was reconstructed with split thickness skin grafts and elevated skin flaps (Figure 2E,F). A wound dressing was applied, and careful attention was paid to protecting the vascular pedicle from pressure.

\section{4 | RESULTS}

The flap sizes ranged from $5 \times 7 \mathrm{~cm}$ to $8 \times 12 \mathrm{~cm}$. Only one superficial vein was anastomosed in each flap. No bone or tendon tissue was added to VS-PIAF flaps. The mean operation time from the first incision to the last skin closure suture was 150 minutes. The patients were encouraged to keep their hands elevated for the first three postoperative days. The patients were discharged at the postoperative third day. In this period, flaps monitored routinely with checking the capillary refill time. A volar splint blocking wrist flexion was used for post-operative 2 weeks. All the flaps have survived completely, and no venous problems or pincushion effects have been seen. The donor
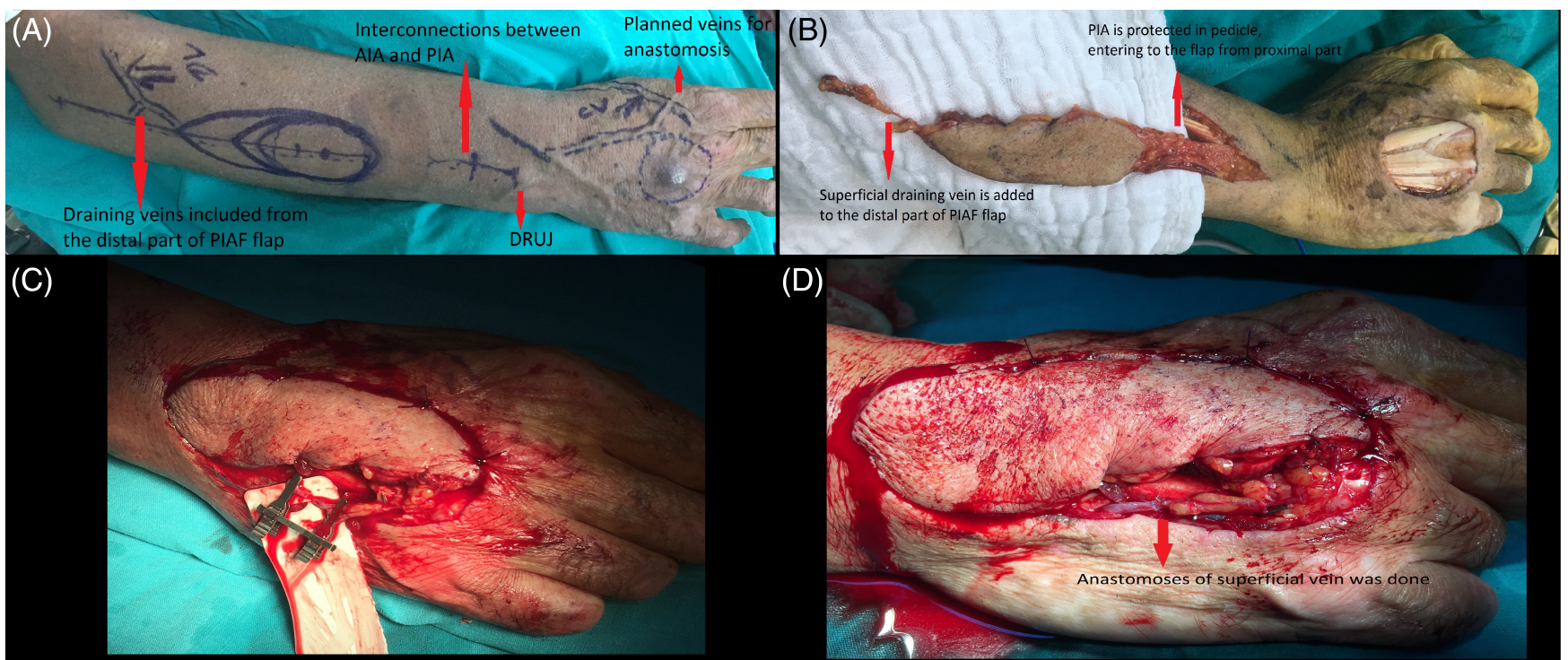

(D)

(E)
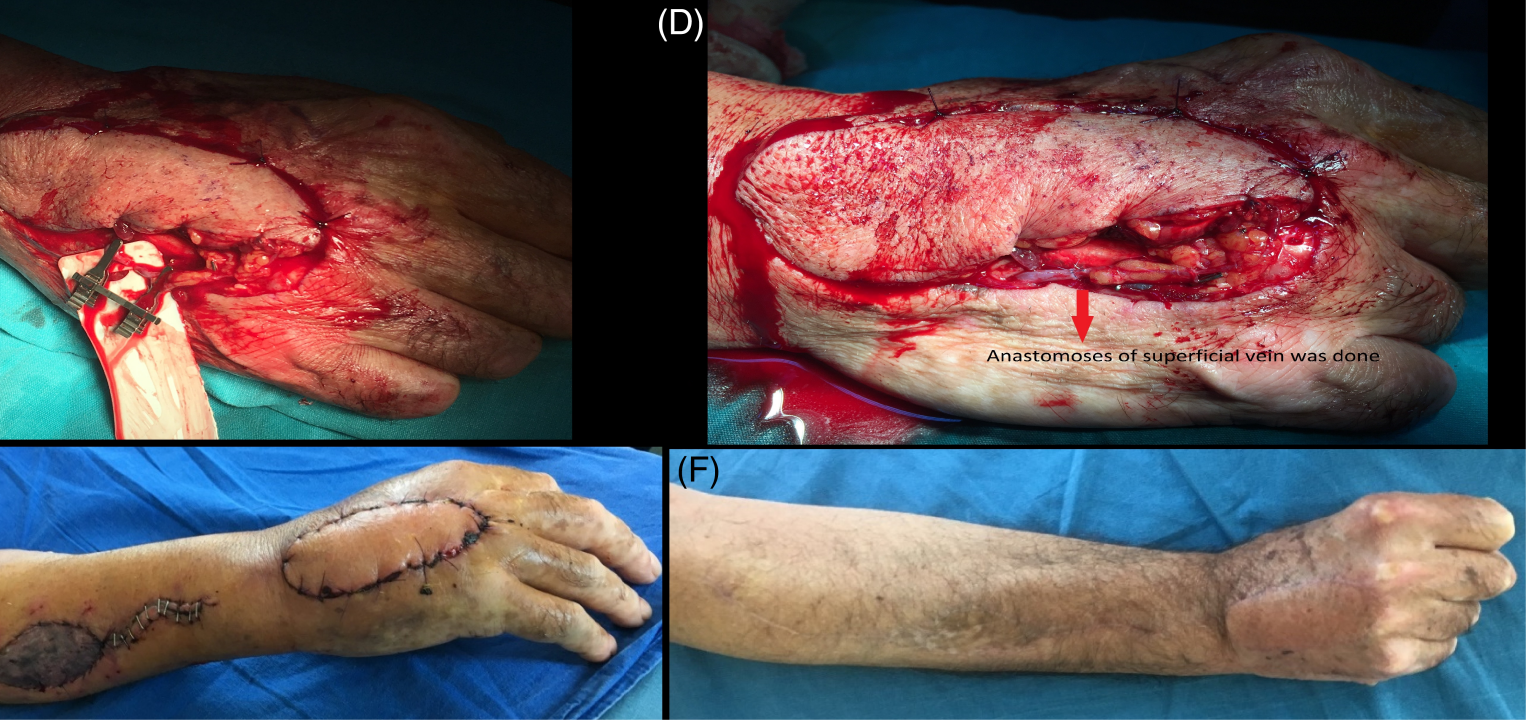

FIGURE 2 A, The operation plan: VS-PIAF and tumor excision markings were outlined. B, The tumor was excised, and the VS-PIAF was elevated with a superficial vein. C, The VS-PIAF was adapted and veins were prepared for anastomosis. D, Superficial vein anastomosis was completed. E, The early postoperative result: the flap donor area was covered with a split thickness skin graft. F, The late postoperative result: healing was completed without any complications, and hand motions were preserved 
areas which have been skin grafted also healed uneventfully, with no early or late complications being observed. Return to work times were variable due to the cause of the defect but the mean return to work time was 5.3 weeks. The mean follow-up time was 15.3 months range 6-26 months. No objective test for final outcome has been performed but both the patient and our satisfactory levels were high and no functional restrictions were seen.

\section{5 | CASE REPORTS}

\section{1 | Case 1}

A 47-year-old male patient had an ulcer on the ulnar side of his left hand. His hand had been burned with hot water when he was a child. $\mathrm{He}$ had hypertension that was under control with medication. An incisional biopsy was made with a pre-diagnosis of Marjolin's ulcer and squamous cell carcinoma was reported. No lymphadenopathy was detected in the antecubital and axillary regions in both clinic and ultrasonography examinations. Simultaneous excision of the tumor and reconstruction with a VS-PIAF was planned (Figure 3A). The tumor was excised with $2 \mathrm{~cm}$ clear margins. As extensor tendons of the hand were exposed without paratenons, a $5 \times 13 \mathrm{~cm}$ VS-PIAF was elevated preserving a proximal superficial vein (Figure 3B). An appropriate superficial vein was dissected on the dorsum of the hand, and venous anastomosis was performed under loop magnification (Figure $3 \mathrm{C}$ ). The flap donor area was covered with a split thickness skin graft, and no complications were seen in the early postoperative period (Figure 3D).

\subsection{Case 2}

A 53-year-old male patient had an ulcerated tumor on the dorsum of his right hand. He had diabetes mellitus and hypertension as comorbidities. An incisional biopsy was made and squamous cell carcinoma was reported. No lymphadenopathy was detected both clinically and with ultrasonograpically in the antecubital and axillary regions. Simultaneous excision of the tumor and reconstruction with a VS-PIAF was planned (Figure 4A). The tumor was excised at least $2 \mathrm{~cm}$ clear margins, that results with a defect including tendons without paratenons. A VS-PIAF with dimensions of $6 \times 9 \mathrm{~cm}$ was elevated preserving a proximal superficial vein. An appropriate superficial vein was dissected on the dorsum of the hand, and venous anastomosis was performed under loop magnification. Primary repair was done on the flap donor area, and no complications were seen in the early postoperative period (Figure 4B). The late postoperative result was satisfactory for both the patient and the surgeon (Figure 4C,D).

\section{6 | DISCUSSION}

The PIAF is widely used in the reconstruction of upper extremity defects. It is a type $B$ fasciocutaneous reverse flap that relies on vascular interconnections between the anterior and posterior interosseous arteries at the distal forearm level (Lamberty \& Cormack, 1990). This flap supplies soft and pliable skin that matches the texture and color of the dorsum of the hand (Zhang et al., 2013). Distally based PIAF are generally used to cover defects of the dorsum of the hand and wrist, but modifications can be made so that defects of the distal interphalangeal joint can be reconstructed (Puri, Mahendru, \& Rana, 2007). Reverse flow PIAFs can be planned for sizes that are as large as $21 \times 10 \mathrm{~cm}$ (Fong \& Chew, 2014). By incorporating a vascularized ulna bone graft or vascularized tendon graft extensor digiti quinti or extensor carpi ulnaris, PIAFs can be used as composite flaps (Lu et al., 2007). Because of the numerous advantages these flaps offer, they have become popular among hand surgeons. The main advantage of this flap is that it protects the major vessels of the hand. While the other common local flaps such as radial and ulnar forearm flaps sacrifice these major arteries, the PIAF is elevated as a reverse flow flap using the perforating arteries of the posterior interosseous artery. Because it is a local flap, operations can be done with an axillary block, with no need for general anesthesia. The skin of the dorsal side of the forearm is thinner than the volar side and supplies a very pliable tissue for the coverage of defects. The constant anatomy of the posterior

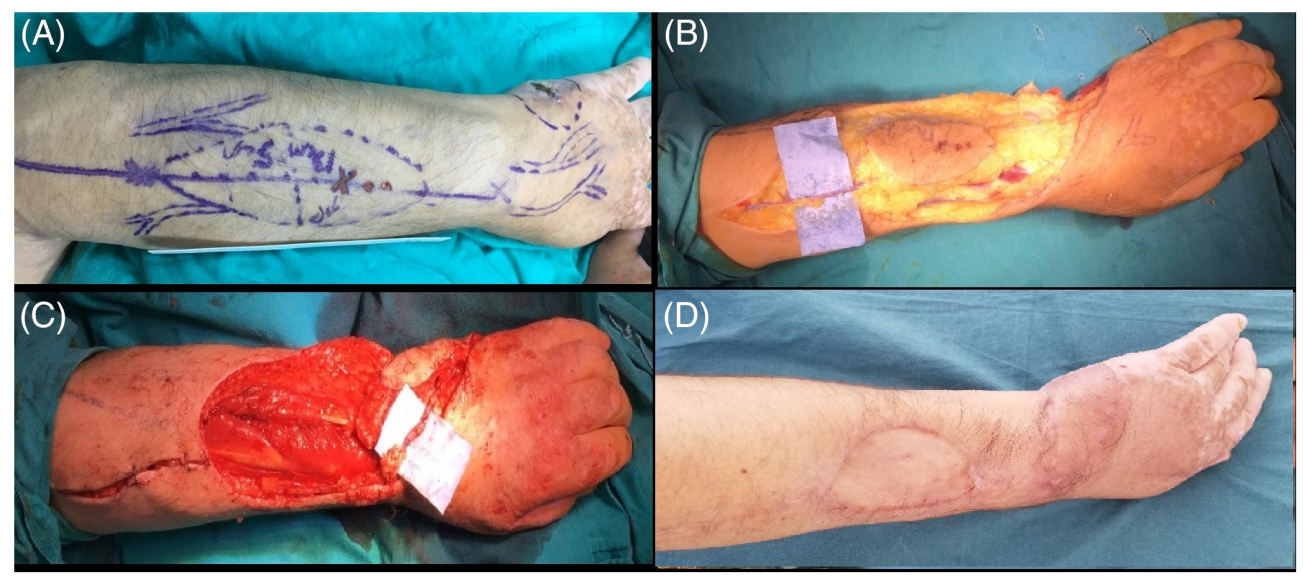

FIGURE 3 A, The operation plan: VS-PIAF and tumor excision markings were outlined. B, The tumor was excised, and the VS-PIAF was elevated with a superficial vein. C, The VS-PIAF was adapted, and superficial vein anastomosis was completed. D, The late postoperative result: healing was completed without any complications 


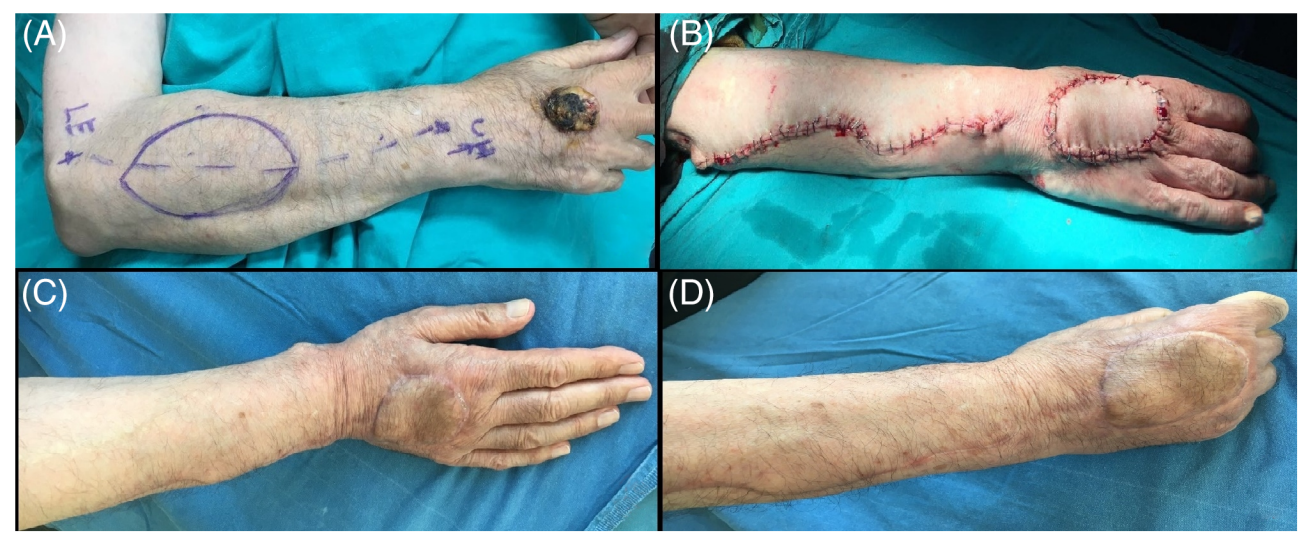

FIGURE 4 A, The operation plan: VS-PIAF markings were outlined. B, The early postoperative result: primary repair was done on the lap donor area. C, The late postoperative result: healing was completed without any complications. D, The late postoperative result: the patient was able to flex his fingers without any tension

interosseous artery and its perforators make the operation relatively easy to perform and the learning period very short. Although some authors suggested to look for the posterior interosseous artery before elevating the flap we elevated the flap first and did not encounter any problems regarding to nourishing vessels (Brunelli et al., 2001). However, the PIAF has some disadvantages, including the fact that being a local flap limits its usage in some cases in which the trauma zone includes distal radio-ulnar joint DRUJ area where the vessels that nourish the flap is found. The major disadvantage with the PIAF is that, because it is a reverse flow flap, venous congestion can occur. Venous insufficiencies cause flap viability problems at least as frequently as arterial insufficiencies do. Several authors mention the venous congestion problems associated with PIAFs. Although some authors, including Zancolli and Puri, report no major complications such as flap failure or venous congestion (Puri et al., 2007; Zancolli \& Angrigiani, 1988), several report higher complication rates. Brunelli et al. report that $15 \%$ of cases had venous congestion and $13 \%$ had ischemic flap necrosis (Brunelli et al., 2001). In addition, Fong et al. present their case series with $21 \%$ of cases having venous congestion that resulted in partial flap necrosis (Fong \& Chew, 2014). Numerous methods have been proposed to overcome venous insufficiency with PIAFs. Ozalp et al. have proposed adding a superficial vein to the pedicle (Ozalp et al., 2016), and Acharya et al. have suggested some modifications, such as creating a broad pedicle with a cutaneous handle and avoiding tunneling for the inset (Acharya et al., 2012). Chen et al. used a wide base to incorporate both branches from the anterior and posterior interosseous arteries in elderly patients and performed additional venous anastomoses in cases where venous congestion was identified after the flap inset (Chen et al., 1998). Using a broad pedicle will enhance the venous return because the wider pedicle does not twist as easily as a thin one, but the cost is a reduction in pedicle mobility. Using venous anastomosis instead of widening the pedicle does not reduce flap mobility, but enhances the venous return. Dissection of superficial veins in the distal part of the flap is a relatively easy procedure to perform, and these veins have similar diameters approximately $2 \mathrm{~mm}$ to those of the dorsal veins of the hand. Also, microvascular end-to-end venous anastomosis can be done under loupe magnification, which is not as time consuming as using a microscope. Although making a venous microvascular anastomosis does prolong the operation time, the benefits are certainly worth it. The use of anterograde venous drainage was proved to be successful for some other reverse flow flaps such as reverse radial forearm flap (Wu \& Brown, 2004). Without venous congestion, the flap tissue is incorporated into the area of the wound in a shorter time and fewer complications are seen, which will surely reduce both hospitalization times and costs. However, another drawback to using this flap is the risk of donor site morbidity when the large flap is elevated. Zhang et al. propose a bi-paddle modification to overcome such donor site morbidity (Zhang et al., 2013) and have used this technique successfully to cover multiple defects. We did not encounter any donor site morbidity in our case series, and the patients presented in this report did not have any multiple defects that required bi-paddle modification. Although we had venous problems in previous cases without this modification, in the present report, no complications were seen in any patients and all the patients were discharged on the third postoperative day. The use of a superficial vein anastomose to overcome venous congestion problems in PIAF as a routine procedure is a novel approach. And this technique may be an alternative method to enhance PIAF survival.

Nevertheless, some negative aspects have been identified in this report. The major disadvantage in using this method is the need for microsurgical skills and equipment. Venous anastomosis is technically more demanding and more prone to occlusion than arterial anastomosis. Incorporating the flap while also aligning the veins requires some experience. Also, the operation is longer because the veins are dissected on both sides and the venous microvascular anastomosis is time-consuming. No tests were done to quantify the patient satisfaction.

\section{7 | CONCLUSION}

The PIAF has been used for reconstruction of upper extremity defects with the advantages of supplying a pliable tissue and without sacrificing major arteries of hand. When used for reconstruction of hand defects as a reverse flow flap, venous congestion problem is the commonly seen problem need to be solved in the early postoperative 
period. Although adding a simple venous microvascular anastomosis will prolong the surgery time, it will overcome some complications such as venous congestion. VS-PIAF is a robust flap that is easy to perform for reconstruction of hand defects and has a short learning curve.

\section{ACKNOWLEDGMENT}

We thank Murat Tuzuner for the schematic illustrations.

\section{CONFLICT OF INTEREST}

The Authors declare that they have no conflict of interest.

\section{ORCID}

Ersin Aksam (D) https://orcid.org/0000-0003-3123-0397

\section{REFERENCES}

Acharya, A. M., Bhat, A. K., \& Bhaskaranand, K. (2012). The reverse posterior interosseous artery flap: Technical considerations in raising an easier and more reliable flap. The Journal of Hand Surgery, 373, 575-582.

Balakrishnan, G., Kumar, B. S., \& Hussain, S. A. (2003). Reverse-flow posterior interosseous artery flap revisited. Plastic and Reconstructive Surgery, 1117, 2364-2369.

Brunelli, F., Valenti, P., Dumontier, C., Panciera, P., \& Gilbert, A. (2001). The posterior interosseous reverse flap: Experience with 113 flaps. Annals of Plastic Surgery., 471, 25-30.

Chen, H. C., Cheng, M. H., Schneeberger, A. G., Cheng, T. J., Wei, F. C., \& Tang, Y. B. (1998). Posterior interosseous flap and its variations for coverage of hand wounds. The Journal of Trauma., 453, 570-574.
Dogra, B. B., Singh, M., Chakravarty, B., \& Basu, S. (2006). Posterior interosseous artery flap for hand defects. Medical Journal, Armed Forces India., 621, 33-35.

Ege, A., Tuncay, I., \& Ercetin, O. (2003). Posterior interosseous artery flap in traumatic hand injuries. Archives of Orthopaedic and Trauma Surgery, 1237, 323-326.

Fong, P. L., \& Chew, W. Y. (2014). Posterior interosseous artery flap: Our experience and review of modifications done. Hand Surgery, 192, 181-187.

Lamberty, B. G., \& Cormack, G. C. (1990). Fasciocutaneous flaps. Clinics in Plastic Surgery., 174, 713-726.

Lu, L. J., Gong, X., Lu, X. M., \& Wang, K. L. (2007). The reverse posterior interosseous flap and its composite flap: Experience with 201 flaps. Journal of Plastic, Reconstructive \& Aesthetic Surgery, 608, 876-882.

Ozalp, B., Elbey, H., Aydin, A., \& Ozkan, T. (2016). Distally based subcutaneous veins for venous insufficiency of the reverse posterior interosseous artery flap. Microsurgery, 36(5), 384-390.

Puri, V., Mahendru, S., \& Rana, R. (2007). Posterior interosseous artery flap, fasciosubcutaneous pedicle technique: A study of 25 cases. Journal of Plastic, Reconstructive \& Aesthetic Surgery, 6012, 1331-1337.

$\mathrm{Wu}$, T. Y., \& Brown, R. E. (2004). Antegrade venous drainage in a reverse radial forearm flap. Plastic and Reconstructive Surgery, 1132, 645-648.

Yazar, S., Chen, H. C., \& Mardini, S. (2008). Augmentation of venous drainage by a venous anastomosis for pedicled flaps. Journal of Reconstructive Microsurgery., 245, 369-376.

Zancolli, E. A., \& Angrigiani, C. (1988). Posterior interosseous Island forearm flap. Journal of Hand Surgery., 132, 130-135.

Zhang, Y. X., Qian, Y., Pu, Z., Ong, Y. S., Messmer, C., Li, Q., et al. (2013). Reverse bipaddle posterior interosseous artery perforator flap. Plastic and Reconstructive Surgery., 1314, 552e-562e.

How to cite this article: Sönmez E, Aksam E, Durgun M, Karaaslan O. Venous super-drained posterior interosseous artery flap for dorsal hand defects. Microsurgery. 2018;38: 876-881. https://doi.org/10.1002/micr.30381 